\title{
Linx
}

Revue des linguistes de l'université Paris X Nanterre

$79 \mid 2019$

Enseigner et apprendre à interagir en langue étrangère : réflexions linguistiques et didactiques

\section{Interaction orale et coopération : un apprentissage interdépendant}

Spoken interaction and peer cooperation: An interdependent learning process

\section{Pascale MANOÏLOV}

\section{OpenEdition}

\section{Journals}

Édition électronique

URL : http://journals.openedition.org/linx/3784

DOI : $10.4000 /$ linx.3784

ISSN : 2118-9692

Éditeur

Presses universitaires de Paris Nanterre

Référence électronique

Pascale MANOÏLOV, «Interaction orale et coopération : un apprentissage interdépendant », Linx [En ligne], 79 | 2019, document 8, mis en ligne le 30 décembre 2019, consulté le 07 avril 2020. URL : http:// journals.openedition.org/linx/3784; DOI : https://doi.org/10.4000/linx.3784

Ce document a été généré automatiquement le 7 avril 2020.

Département de Sciences du langage, Université Paris Ouest 


\title{
Interaction orale et coopération : un apprentissage interdépendant
}

Spoken interaction and peer cooperation: An interdependent learning process

\author{
Pascale MANOÏLOV
}

\begin{abstract}
«We do not learn from experience... we learn from reflecting on experience. » (John

Dewey 1933)
\end{abstract}

\section{Introduction}

1 Parmi les activités langagières travaillées en classe de langue, l'interaction orale est sans doute celle qui est la moins développée et la moins explorée, surtout quand il s'agit d'interactions entre pairs. En effet, en raison d'une mise en œuvre contraignante dans des classes composées de 30 élèves et d'une difficulté certaine dans la collecte de données empiriques, la recherche ne s'est encore que peu penchée sur le discours-eninteraction tel qu'il est produit par des apprenants en milieu institutionnel. Cet article rend compte d'une recherche-action dont l'objectif était de collecter et analyser des interactions de classe entre pairs afin d'en faire émerger les spécificités. A partir de ces données, un dispositif de formation visant l'apprentissage de et dans l'interaction orale en langue vivante étrangère (dorénavant LVE) a été conçu et mis en place dans plusieurs classes. Les échanges qui se déroulent en interaction orale ont la spécificité d'être co-construits (Lazaraton \& Davis 2008), autrement dit, l'avancée de la conversation dépend de ce que chaque locuteur dit mais également de la manière dont il tient compte ou rebondit sur ce qui a été dit avant et ce qu'il anticipe des interventions à venir. Cette activité langagière requiert donc des participants qu'ils sachent co-opérer (Goodwin 2017) en LVE afin de garantir la cohérence et la cohésion de leur discours-en-interaction pour atteindre ensemble leur but.

Cet article adopte une double perspective didactique et interactionniste. La première permet de proposer une réflexion sur les concepts qui ont prévalu lors de la conception d'un dispositif pédagogique destiné à favoriser le développement de la compétence 
«apprendre à apprendre » (Holec 1990). La seconde, quant à elle, vise l'analyse des effets de l'approche pédagogique sur les apprenants. On se place dans un premier temps dans un paradigme d'enseignement puis dans un second temps, dans un paradigme d'apprentissage. Car former les élèves à l'interaction orale suppose de leur permettre d'apprendre en interagissant, mais n'est-il pas nécessaire, avant même d'engager ce projet, de leur permettre d'apprendre à interagir?

Cet article sera l'occasion de poser un autre regard sur ces questions. En effet, le niveau de maturité et d'autonomie des adolescents n'est pas encore suffisant chez tous ${ }^{1}$ pour espérer qu'ils évaluent seuls leurs besoins et prennent toutes les mesures nécessaires à la mise en place de stratégies d'apprentissage (Tarone 1980) ou d'autorégulation.

La première partie de l'article ainsi sera l'occasion d'interroger les notions d'autonomie, de collaboration et de coopération ainsi que la question de la métacognition dans une perspective de développement de la compétence d'interaction orale. Puis les actions de formation visant un apprentissage stratégique de l'interaction orale seront présentées ainsi qu'une analyse du discours de quelques apprenants qui sont engagés dans un dispositif visant à prendre conscience de leurs réussites et de leurs besoins avec l'aide de leurs pairs.

\section{Présentation du projet}

5 Le projet porte sur la formation à la compétence d'interaction orale d'élèves de $3^{\text {ème }}$ (dernière année de collège, âge 14/15 ans) de niveau A1+ à A2+ (Conseil de l'Europe 2001), dans quatre classes d'anglais en France et, de mesurer les effets de cette formation. Celle-ci a consisté dans un premier temps en une présentation et une réflexion sur les stratégies propres à faciliter et développer les échanges entre pairs. Puis tout au long de l'année, lors de séances où les élèves travaillaient en interaction orale, des activités de formation ciblées ont été proposées. Le but était alors de susciter une réflexion métacognitive.

\section{Les concepts clés}

\subsection{L'interaction entre pairs}

Philp (2014: 3) et ses collègues définissent l'interaction entre pairs comme «toute activité de communication entre apprenants, dans laquelle la part d'intervention de l'enseignant est réduite ou nulle $»^{2}$ (voir également Oursel et Manoïlov 2020). Autrement dit, ce sont des échanges horizontaux puisque les élèves sont placés en groupe d'au moins deux participants qui communiquent de façon autonome. Ce statut d'élève implique une relation symétrique basée sur une certaine homogénéité d'âge, d'expérience scolaire et d'habitudes de travail qui sont spécifiques à un pays comme la France. En classe, l'interaction entre pairs est ainsi l'occasion de vivre une expérience sociale partagée qui inscrit les apprenants dans un contexte commun.

7 Apprendre la langue dans l'interaction est effectivement un moyen pour les apprenants d'être actifs, à la fois en tant que producteur d'un discours mais également en tant qu'interprète du discours de l'autre. Néanmoins la densité (c'est-à-dire le temps et la fréquence) de pratique nécessaire pour permettre l'acquisition d'un discours-en- 
interaction qui soit fluide et performant est considérable (voir notamment Hilton 2019). Les apprenants d'une LVE, lorsqu'ils étudient en milieu institutionnel exolingue, ne disposent pas d'un temps d'exposition similaire à celui dont ils ont bénéficié lors de l'acquisition de leur langue maternelle ou de leur langue de scolarité. En revanche, ils ont acquis une maturité cognitive bien supérieure à celle d'un nourrisson et sont déjà des locuteurs compétents dans cette même langue. Cette compétence est un atout sur lequel il est possible de capitaliser et de s'appuyer pour asseoir des stratégies d'autorégulation. Pour Jézégou (2013: 184) l'autorégulation se « réfère au contrôle que l'apprenant exerce sur son processus cognitif d'apprentissage, en anticipant, préparant, évaluant et ajustant ses procédures en fonction des effets ou des résultats observés ». Afin d'exercer un contrôle, il est nécessaire que l'apprenant ait conscience des stratégies qui facilitent l'apprentissage ainsi que des moyens à mettre en œuvre pour s'approprier ces stratégies. Construire un discours-en-interaction avec un pair peut ainsi être l'occasion de fixer des objectifs, de réfléchir aux méthodes les plus appropriées pour les atteindre et durant les échanges, de prendre conscience de ses réussites ou de ses besoins (Holec 1991). Le travail entre pairs peut également permettre d'aller plus loin en donnant l'occasion aux apprenants d'engager une réflexion métacognitive collective reposant sur des échanges peu menaçants pour la face, et cela en raison de la symétrie de statut. En abaissant les risques socio-affectifs ${ }^{3}$, la confiance et la motivation sont alors susceptibles d'augmenter.

Les dispositifs pédagogiques peuvent alors aider les apprenants à mettre en place des stratégies métacognitives, d'interaction ou de médiations. A la suite d'Anderson (1987), Bange (2005 : 37) insiste sur la dimension cognitive de la maitrise de savoir-faire ou " savoir procédural » c'est-à-dire l'intelligence opérative d'un individu qui lui permet de " savoir comment plutôt que simplement savoir que » (Bruner 1983 : 255). Autrement dit, on oppose savoir procédural à savoir déclaratif, ce dernier étant tout le savoir sur les faits, toute l'expérience que nous avons emmagasiné en mémoire et que nous pouvons verbaliser. Dans le cas de l'apprentissage d'une langue vivante, cela se rapporte aux règles grammaticales, aux connaissances lexicales et phonologiques ainsi qu'à toutes les connaissances sociolinguistiques ou pragmatiques. Le passage d'un savoir (déclaratif) à un savoir-faire (savoir procédural) requiert de l'apprenant un effort considérable (Anderson ibid.) qui repose sur deux prémisses distinctes identifiée par Bange (2005) : la prémisse intentionnelle et la prémisse cognitive. À partir d'une volonté individuelle d'agir sur une situation pour la modifier (prémisse intentionnelle), l'apprenant met en œuvre un processus cognitif (prémisse cognitive) qui lui permet de traiter le problème rencontré, grâce notamment au répertoire de moyens qu'il a acquis au cours de son expérience. C'est l'adéquation entre les opérations de résolution du problème et le désir d'action émergeant de l'analyse initiale de la situation qui permet le glissement du savoir déclaratif en un savoir-faire en développement. Mais la description de ce processus sous-entend une certaine circularité : comment peut-on accomplir une action ou mettre en œuvre un comportement qu'on est justement censé apprendre? Bange (ibid.) qui s'appuie sur les théories de Vygotsky et Bruner, voit la résolution de ce paradoxe dans l'interaction. C'est grâce à la médiation d'un autre acteur participant à l'interaction qu'un étayage peut être mis en place par exemple. Selon Bruner, l'apprentissage correspond au passage de la régulation du comportement par autrui à une régulation autonome, c'est-à-dire que la modification de la compétence individuelle passe par une action conjointe en interaction avec des partenaires. 


\subsection{La coopération et la collaboration entre pairs}

Le développement des activités d'interaction orale entre pairs implique que les participants développent une posture favorable au travail en groupe ${ }^{4}$ et sous-entend ainsi les notions d'apprentissage coopératif et d'apprentissage collaboratif.

Les deux termes sont souvent considérés comme synonymes en ce qu'ils impliquent à la fois un travail en groupe et le fait que les participants aient un but commun visant une réalisation collective, comme une tâche ou un projet ( D. W. Johnson and R. T. Johnson 1990). En ce sens, les interactions entre pairs, par opposition aux interactions élèves/ enseignant ou natif/non-natifs, seraient idéales pour susciter des apprentissages. Néanmoins, la conception et les principes de fonctionnement des modes coopératifs ou collaboratifs varient sur un certain nombre de points majeurs (Baudrit 2007) qui sont susceptibles d'impacter les conditions d'apprentissage dans et de l'interaction orale.

\section{a. Le concept de coopération}

L'apprentissage coopératif trouve son origine dans la psychologie sociale américaine (D. W. Johnson and R. T. Johnson 1974, 1975 ; Slavin 1977) et le travail en groupes coopératifs s'inspire des techniques d'apprentissage de diverses disciplines pour permettre aux élèves de travailler et surtout d'apprendre ensemble de façon autonome. Les principes régissant ce type de groupes reposent sur une structuration hétérogène du groupe par l'enseignant, qui initie les apprenants au travail collectif, les sensibilise à l'entre-aide et prévoit une répartition des rôles pour l'atteinte des objectifs. De cette organisation, il résulte une interdépendance entre les membres du groupe et de ce fait, une responsabilisation de chacun, toute défaillance ayant une implication sur les résultats de l'équipe.

Les programmes allant de méthodes d'apprentissage structurées en équipes, telles que le student team learning (STL) développés par l'université J. Hopkins aux Etats-Unis jusqu'aux méthodes d'apprentissage informel en groupe telles que le dispositif Jigsaw de E. Aronson et al. (1978) ou le Learning together ( D. W. Johnson and R. T. Johnson 1975, 1990) ont mis en avant les bénéfices de ces organisations de groupe. Calderón et al. (2011) rappellent que la structure la plus efficace telle qu'elle a été observée aux EtatsUnis chez des apprenants hispanophones de l'anglais est le travail en groupe coopératif composé de 4 élèves qui s'enseignent ce qui a été appris en classe.

Selon Slavin (1996), quatre facteurs viendraient nourrir le travail coopératif :

- la motivation qui serait le facteur le plus important favorisant les processus d'apprentissage ;

- la cohésion sociale (également appelée théorie de l'interdépendance) qui postule que les apprenants s'entre-aident dans l'apprentissage, chacun bénéficiant de l'appartenance au groupe (esprit de groupe) pour sa construction identitaire personnelle ( D. W. Johnson et R. T. Johnson 1998) ;

- les théories sociocognitives telles que développées par Vygotsky (1978) qui portent sur l'aspect développemental et s'appuient sur l'asymétrie de compétences entre les partenaires;

- les théories sociocognitives qui portent sur la dimension cognitive telle que décrite en psychologie cognitive. Dans cette perspective, il est postulé que pour que l'information soit inscrite dans la mémoire et reliée aux données déjà mémorisées, l'apprenant doit opérer une restructuration cognitive de ce qu'il a déjà mémorisé 
(1998). Le moyen le plus efficace pour mener ces opérations étant de devoir expliquer ce qu'on a appris à une autre personne car c'est la restructuration ou l'élaboration de nouvelles ressources qui favorisent les apprentissages. levier de motivation pour l'apprentissage car il favorise des facteurs comme l'orientation vers un but, la cohésion du groupe, l'autonomie de l'apprenant, la confiance en soi et l'intérêt intrinsèque. L'autonomie dont jouissent les participants serait la dimension la plus importante du travail coopératif. La responsabilisation serait susceptible de stimuler un investissement soutenu dans la durée, par opposition aux situations contrôlées par l'enseignant qui favorisent davantage un investissement ponctuel car plus ou moins contraint.

\section{b. Le concept de collaboration}

15 Quant au concept de collaboration, il trouve ses origines à la fois dans la perspective piagétienne (Damon 1984) mais également celle de Vygostsky (1978). Il repose sur les habiletés et les contributions des membres du groupe (Panitz, 1999) et s'appuie sur les travaux de Dewey (1933) qui voyait l'éducation comme une entreprise sociale où chacun peut participer et se responsabiliser (Baudrit 2007).

Les groupes collaboratifs ont une organisation différente de celle des groupes coopératifs: le principe est la liberté d'organisation qui doit susciter des échanges libres, non dirigés. Ceux-ci s'appuient sur le caractère symétrique des places et des positions occupées, ce qui participe à l'équilibre du groupe et est ainsi susceptible de soutenir les échanges et les débats d'idées. Dillenbourg (1999) considère d'ailleurs cette dimension comme indispensable au bon fonctionnement de la collaboration. C'est le mode de fonctionnement du travail collaboratif qui est signifiant ici puisque les discussions qui peuvent s'engager dans ces groupes sont susceptibles de faire émerger des différences d'idées, de conception ou dans le cas particulier des langues vivantes, de résolution de problèmes d'intercompréhension ou d'intersubjectivité. Ces divergences sont alors déclencheurs de ce que les psychologues sociaux de Genève identifient comme des conflits socio-cognitifs (Doise et Mugny 1981, Perret-Clermont 1979) qui jouent «le rôle de perturbateurs à l'origine de la reconstruction intellectuelle » (Baudrit 2007 : 111).

\section{c. Coopération versus collaboration : quel schéma adopter en LVE ?}

17 Des travaux (voir notamment Grist 2009, Mercer 2013), adoptant l'hypothèse du 'cerveau social', ont étudié la question de l'apprentissage en groupe et la résolution de problèmes. Des recherches, de type interventionniste, se sont également attachées à mettre en place des dispositifs visant l'amélioration des relations sociales et de la communication entre les pairs afin d'apprécier l'éventuelle amélioration de la qualité des apprentissages réalisés en collaboration. Certaines mettent l'accent sur le discours qui précède ou suit la réalisation de la tâche. Howe (2010) rend compte de plusieurs études dans lesquelles des élèves doivent prédire, dans le cadre d'une discussion entre pairs, les résultats supposés de diverses expérimentations en sciences puis rendent compte de leurs observations. Leur compréhension des phénomènes est mesurée à l'aide de pré et post-tests. Il s'avère que les résultats sont meilleurs lorsque (a) il a été demandé aux élèves de rechercher un accord sur la prédiction avant de mener l'expérience et (b) lorsque les élèves ont exprimé des avis contradictoires lors des 
discussions. Selon l'auteur, ces résultats supérieurs seraient dus, d'une part au fait que devoir se mettre d'accord engage les élèves dans des discussions plus approfondies et donc des conclusions plus élaborées, d'autre part au fait que les contradictions non résolues amorcent une réflexion métacognitive sur leurs hypothèses et la signification de leurs observations. Lorsque ce type d'approche est transposé dans la classe de LVE, cela implique de proposer une phase de préparation (Manoïlov 2019) ou de planning (Ellis 2003), puis après la tâche une phase pendant laquelle l'observation des méthodes mises en œuvre seront discutées et évaluées pour souligner les processus métacognitifs développés ou à développer.

Baudrit (2007), dans son analyse comparative de l'apprentissage coopératif et de l'apprentissage collaboratif, semble envisager les deux dispositifs comme pouvant répondre à des besoins précis selon le stade de développement : là où on s'attache à fixer des apprentissages fondamentaux, l'apprentissage coopératif, qui implique un contrôle plus grand de l'enseignant, serait plus pertinent, tandis que pour des activités visant le développement de l'esprit critique, du raisonnement ou de l'exercice du jugement, la mise en retrait de l'enseignant est plus légitime, ce qui s'applique dans l'apprentissage collaboratif.

On peut donc envisager ces deux types de dispositifs sur un continuum avec à une extrémité une modalité de travail très structurée et assortie d'un cadrage assez serré par l'enseignant pour aller vers une construction des interactions laissée davantage à l'initiative des apprenants et des interventions de l'enseignant plus orientées vers un guidage réflexif. Dans le cas des interactions orales en classe de LVE, les activités focalisées sur la forme seraient ainsi davantage axées sur la coopération tandis que celles axées sur le sens, avec une orientation plus collaborative. La première peut ainsi être un préalable à la seconde. Par ailleurs, le mode coopératif peut permettre d'apprendre à travailler ensemble puis le mode collaboratif peut prendre le relais pour des tâches plus complexes.

\subsection{L'autonomie}

Placer les apprenants en situation d'interaction entre pairs (en mode coopératif ou collaboratif) implique de leur accorder une autonomie d'action puisque l'enseignant n'est plus là pour réguler les échanges. En effet, les classes de collège en France sont généralement composées de 24 à 32 élèves (MENJ 2019), ce qui signifie que 12 à 16 binômes travaillent simultanément à mener des activités d'interaction orale.

21 Les enfants, dès leur plus jeune âge, déploient leur agentivité (Bandura 1989) c'est-àdire leur capacité à agir pour atteindre les buts qu'ils se fixent. Le petit enfant qui apprend à parler montre par exemple un objet pour désigner ce à quoi il réfère et obtient alors de l'adulte une forme verbale. Ces formats transactionnels qui passent par des expressions mimo-gestuelles conjuguées ou non à des formes verbales adressées à l'adulte sont autant d'occasions pour l'enfant, dans l'interaction, d'agir sur le monde qui l'entoure et apprendre la langue avec un locuteur compétent. Le processus d'apprentissage se poursuit tout au long de l'enfance puis de l'adolescence. Or, en milieu institutionnel, les élèves sont assis en classe derrière leur table et c'est l'enseignant qui détermine quoi et quand apprendre. Le « comment apprendre » est en général assez peu développé, quant au pourquoi, il relève de l'implicite et n'émane que rarement des objectifs que se forment les apprenants. Mon expérience d'enseignante 
dans le secondaire m'a permis d'observer que si certains adolescents ont la chance d'avoir des projets et des envies, pour nombre d'entre eux, l'avenir apparaît très flou et assez anxiogène. Les premiers parviennent à faire un lien avec les enseignements qui leur sont proposés et donc à s'engager dans les apprentissages, tandis que les seconds restent souvent passifs. Le cadre très guidé du collège laisse en effet peu d'espace pour susciter leur investissement et leur engagement personnel. Cela a pour conséquence une certaine hétérogénéité des conduites d'apprentissage (Arditty et Vasseur 2005). C'est pourquoi la question de l'autonomie des élèves est vitale car elle envisage un renversement de la responsabilité d'apprentissage souvent perçue comme étant sous le contrôle de l'enseignant. En impliquant les apprenants dans la prise en charge de leurs besoins et l'observation de leurs pairs, ils sont davantage susceptibles de se fixer des objectifs et de se donner les moyens de les atteindre.

L'autonomie des apprenants a été questionnée dès le début des années 1980 (voir notamment Holec 1979, 1991), pour explorer la capacité des apprenants à réaliser seuls un programme (quel qu'il soit), c'est-à-dire sans accompagnement. Dans le cadre de l'apprentissage des langues étrangères, des méthodes en autoformation (par exemple Berlitz ou ASSIMIL) ont eu leur heure de gloire mais sans finalement tenir leurs promesses. A partir des années 90 et à la faveur des approches centrées sur l'apprenant, la conception d'autonomie a évolué en considérant que l'apprenant ne réalise pas nécessairement le travail d'apprentissage de manière isolée mais pour lui-même. La recherche a largement porté à l'époque sur l'étude des attitudes, motivations ou des représentations des apprenants, celles-ci étant considérées comme jouant un rôle central. Van Lier (1996) considère l'autonomie comme un facteur décisif dans le processus d'apprentissage langagier. Cette interprétation est étayée par l'étude de Deci and Ryan (1985).

" The need of autonomy (i.e. the desire to be self-initiating and to regulate one's actions) is an innate human need. Some sense of autonomy is seen as a prerequisite for any behavior to be intrinsically rewarding » (cité par Ehrman \& Dörnyei 1998 : 259)..$^{5}$

L'autonomie est notamment envisagée par opposition au contrôle que peut habituellement exercer l'enseignant sur la direction des échanges et sur la définition de la situation. Pour Little (2007: 15), « la nature même de l'autonomie de l'apprenant est sa capacité de prendre en charge son propre apprentissage ». Il ne s'agit donc plus de laisser l'apprenant seul mais de lui proposer un guidage et des conditions qui favorisent le travail cognitif. Dans une perspective socio-interactionniste, cela implique de permettre à l'élève d'échanger, de partager, de s'entrainer avec ses pairs notamment. C'est dans le cadre de pratiques sociales d'interaction que l'apprentissage pourra être pris en charge par l'apprenant. Il s'agit donc pour l'enseignant d'organiser les conditions qui favoriseront les apprentissages. Holec (1990) distingue trois approches parmi les démarches pédagogiques qui ont pour objectif de développer l'autonomie :

- autonomie signifie indépendance : l'apprenant est "seul sur un matériel adéquat", « hors de la présence d'un enseignant ». Comme indiqué précédemment, ce type de pratique « ne développe que marginalement la capacité de l'apprenant à prendre en charge son apprentissage » (pré-construit) ;

- autonomie signifie exercice actif de sa responsabilité d'apprenant : "c'est une façon d'apprendre qui est centralement visée (apprendre en auto-direction), et l'apprenant apprend à apprendre par contrecoup, implicitement, voire intuitivement »; 
- autonomie signifie capacité d'apprendre: "c'est la capacité d'apprendre qui est centralement visée, et l'apprenant apprend à apprendre ouvertement, explicitement. »

Il faut donc, toujours selon Holec (1990), que l'apprenant s'engage dans une démarche qui relève de sa responsabilité individuelle et qu'il domine. L'usage du verbe « dominer » fait référence à des processus explicites plutôt qu'implicites. Ainsi, on considère que les apprenants sont à l'initiative des actions menées et les contrôlent, et non que cellesci reposent sur un fonctionnement cognitif inconscient et involontaire. Cela implique un double objectif pour l'enseignant qui doit aider l'apprenant à, d'une part atteindre ses objectifs linguistiques et communicatifs, d'autre part s'autonomiser pour construire son apprentissage.

For Holec the concept of learner autonomy has consequences not only for the way in which learning is organised but also for the kind of knowledge that is acquired. If the learner himself determines the goals and content of learning, 'objective, universal knowledge is [...] replaced by subjective, individual knowledge'.6 (Little $2007: 16)$

L'apprentissage (c'est-à-dire ce qui est appris) échappe ainsi au contrôle de l'enseignant qui ne peut plus considérer les connaissances et les savoir-faire comme des objectifs universaux mais doit accepter qu'ils soient subjectifs et propres aux individus (Holec 1990). Dans cette optique, l'enseignant vient alors proposer des activités qui visent l'acquisition d'attitudes et d'habiletés permettant de définir ce qu'il faut apprendre et comment procéder.

La littérature sur le sujet présente l'apprentissage autorégulé (i.e. l'exercice d'un contrôle sur les processus cognitifs) ou autodirigé (i.e. l'exercice d'un contrôle sur les dimensions psychologiques et pédagogiques) comme étant les caractéristiques d'un certain type d'individus.

Individuals [that] take the initiative, with or without the help of others, in diagnosing their learning needs, formulating their learning goals, identifying human and material resources for learning, choosing and implementing appropriate learning strategies, and evaluating learning outcomes. ${ }^{7}$ (Knowles 1975 :

18)

Plusieurs études rendent compte de dispositifs dans lesquels une formation explicite à certaines stratégies a été mise en place : stratégies pour écouter et comprendre (Roussel 2019, Vandergrift and Goh 2012), pour s'exprimer à l'oral (Cohen and Weaver 1998, Dörnyei 1995, Nakatani 2005) et pour lire et écrire (Bianco 2016, Macaro, 2001). Toutes ces recherches montrent que les apprenants qui font un usage conscient de ces stratégies produisent un niveau de performance langagière supérieure à ceux qui sont moins "stratégiques » à ce niveau. On parle alors de métacognition qui est définie par Tobias et Everson comme " la capacité à gérer, évaluer et planifier son apprentissage ${ }^{8}$ (2009: 108). La psychologie développementale s'est emparée de la recherche dans ce domaine et souligne l'importance des processus métacognitifs pour soutenir la motivation et les apprentissages. Le postulat développé par les chercheurs est le suivant: si les étudiants ne parviennent pas à identifier ce qu'ils savent de ce qu'ils doivent encore apprendre, il est peu probable qu'ils mettent en place des stratégies métacognitives telles que l'évaluation de leurs apprentissages ou bien qu'ils développent des stratégies d'apprentissage plus efficaces. Par ailleurs, la distinction entre un apprenant avancé et un plus novice relève bien sûr des connaissances et compétences maitrisées mais également du regard qu'ils portent sur leurs propres apprentissages et sur les stratégies mises en place. A cet égard, l'importance accordée 
aux échanges en classe autour de la métacognition ainsi que de la réflexion menée par les apprenants s'avère être de plus en plus plébiscitée (Whitbread et al. 2013). Les résultats, généralement encourageants, permettent de s'accorder sur l'importance de l'usage du langage; néanmoins, dans le cadre d'échanges en langue étrangère, des limites naturelles s'imposent au travail collaboratif médié par le langage. Selon Jones (2008),

the coordination of collective activity through language presupposes individuals who are capable of using language as a means of organising and controlling their own actions and behaviour within the communal task. (Jones 2008, p.81) ${ }^{9}$

Par ailleurs, la littérature traitant de la question de l'autonomie (voir par ex., Barbot et Camatarri 1999, Rivens Mompean 2013, Château et al. 2015) montre qu'il s'agit de différencier l'autonomie perçue comme un objectif et l'autonomisation qui est le résultat d'un processus par lequel l'apprenant, en s'appuyant sur un cadre et un environnement toujours en mouvance, arrive à développer son autonomie. C'est donc davantage de l'autonomisation des apprenants qu'il sera question ici, car les actions de formation portent notamment sur la mise en place d'un processus d'entrainement, qui à son tour servira des objectifs d'apprentissage.

\section{Le protocole testé}

La recherche-action présentée ici s'appuie sur trois années d'observation filmées d'interactions orales entre pairs qui ont permis de faire émerger certaines spécificités des échanges entre apprenants d'une langue étrangère et notamment, leurs difficultés à co-construire un discours cohésif (Manoïlov 2017). Durant la troisième année, un protocole spécifique a été mis en place. Celui-ci inclut d'une part une formation initiale aux stratégies d'interaction orale, d'autre part des séances tout au long de l'année pendant lesquelles, outre l'entrainement réalisé en binômes, des modules méthodologiques ont permis de sensibiliser les élèves à certaines stratégies. Le projet s'articule autour de l'apprentissage dans l'interaction d'une part, et l'interaction pour apprendre à apprendre d'autre part.

La formation initiale, en début d'année scolaire, a été menée à partir d'extraits vidéo d'interactions entre apprenants francophones de l'anglais en classe. Les élèves ont été invités à repérer les stratégies de réussites (Nakatani 2010) utilisées par les partenaires, chacune étant identifiée, nommée puis assortie de ressources linguistiques pour les mettre en œuvre. Par exemple, les diverses formulations en anglais, utilisables pour demander à son interlocuteur de clarifier son propos, ont été notées. La fiche est complétée tout au long de l'année scolaire. La conscientisation de ces stratégies permet alors aux apprenants de disposer de schémas opératoires adaptés pour la gestion de leurs échanges. Quant au dispositif mis en place au cours de l'année (la formation continue), il comprend à chaque fois une tâche d'entrainement précédée et suivie d'activités de conscientisation du travail sur les stratégies d'interaction. Au cours de certaines séances, des élèves ont pu être filmées et c'est l'une des séances qui est décrite ci-dessous pour illustrer notre propos.

La tâche de déficit d'information (Prabhu 1987) proposée s'inscrit dans une séquence qui permet d'aborder la question de la diversité et du multiculturalisme aux Etats-Unis (thème culturel des Voyages et migrations du programme de cycle $4^{10}$ ). L'étude porte sur un lieu emblématique de l'immigration aux Etats-Unis : Ellis Island. Il s'agit de faire 
découvrir la vie d'un immigrant au début du vingtième siècle, dans une perspective actionnelle. À l'issue de la séquence pédagogique, les élèves doivent rédiger une lettre de récit de voyage. Afin de mener à bien cette tâche finale, une progression raisonnée est proposée avec des tâches intermédiaires faisant appel à diverses activités langagières. La tâche décrite ici est organisée sous forme d'un jeu de rôle: une interview entre un descendant d'immigrant et un journaliste. La phase de préparation est dédiée à un travail de compréhension de l'écrit et de rédaction de questions puis de préparation de l'interaction orale. Ce travail préparatoire a pour objectif de faciliter les échanges et de susciter motivation et engagement chez les élèves. Cette mise en œuvre reprend le modèle de tâche avec préparation, décrite par Manoïlov (2019).

L'objectif principal est le développement des compétences discursives, assorti d'objectifs langagiers et méthodologiques :

- préparer une interaction orale à partir d'un texte écrit : repérer des informations explicites et les comprendre pour pouvoir en parler (élucidation du sensvocabulaire, structures complexes - et réalisation phonologique) ou pouvoir interroger un partenaire pour obtenir des informations ;

- développer des stratégies de réussite et notamment reprendre les propos de l'allocutaire ou les reformuler. Il s'agit dans le premier cas de la reprise écho qui consiste en une reprise partielle ou complète de l'énoncé de l'interlocuteur. Quant à la reformulation, c'est une modification du discours qui change, simplifie ou complexifie un énoncé pour favoriser l'intercompréhension entre les participants.

Pourquoi se focaliser sur la reprise et la reformulation? À défaut d'être significative en termes de création linguistique, la reprise du discours d'autrui «se révèle être une trace subtile des compétences interactives en développement » (Pekarek Doehler et al. 2008: 5). En effet, l'énoncé produit est disponible dans la mémoire immédiate de l'interlocuteur qui peut le reprendre avec un minimum d'effort cognitif (Long and Porter 1985, Pica, 1996). La reprise forme un point d'appui pour l'allocutaire qui dispose dans sa mémoire immédiate de l'énoncé qui vient d'être produit. Elle a donc un rôle structurant qui participe à la cohésion du discours (Berger 2008). Or, lors des interactions orales observées entre les apprenants durant les deux premières années de la recherche, il est apparu que les élèves avaient assez peu recours aux reprises, sauf sous forme de blocs lexicalisés acquis au début de leur apprentissage de la langue. Par exemple: What's your favourite colour? My favourite colour is blue, ce qui donne régulièrement lieu à des transferts maladroits du type: What's your nationality? $M y$ nationality is ...

Le jeu de rôle est également l'occasion de mettre en place une phase d'observation par des pairs qui peuvent alors prendre conscience des méthodes développées par les interlocuteurs (reprises, répétitions, reformulations), ou au contraire qui seraient à développer, ainsi que des stratégies de gain de temps (gestion des pauses et usage de gap-fillers) auxquelles les élèves ont été formés. Cette observation est guidée par une grille fournie par l'enseignante qui permet aux élèves de noter les opérations apparues dans les conversations. Ils font ensuite un retour à de leur partenaire sur les stratégies développées lors des échanges. Le repérage proposé doit guider les apprenants pour qu'ils prennent conscience des opérations spécifiques permettant à la conversation de progresser de manière fluide, c'est-à-dire en préservant cohésion et cohérence.

La mise en œuvre de ces activités a donné lieu à une préparation rigoureuse et un enchainement très progressif : chaque étape s'appuie sur un travail coopératif qui 
participe à une construction de compétences linguistiques, pragmatiques et méthodologiques.

\subsection{Avant l'interaction (phase de préparation) : formation méthodologique aux stratégies de réussite}

Étape 1 : Retour sur la fiche méthodologique sur les stratégies d'interaction, établie en début d'année et rappel des stratégies pertinentes pour la réalisation de la tâche.

Étape 2 : Étude d'une fiche méthodologique d'étape.

Avant le jeu de rôle, une formation méthodologique a été proposée aux élèves pour leur présenter les méthodes et ressources langagières qui s'offrent à eux. Celles-ci se focalisent sur quelques stratégies :

- la reprise des questions ou des informations communiquées : pour gagner du temps, pour vérifier la compréhension ou clarifier la demande ;

- la demande d'aide : faire répéter, demander des explications ou une clarification ;

- le gain de temps: utiliser des gap fillers et des reprises pour prendre le temps

d'élaborer sa réponse.

Étape 3 : Préparation de l'interaction orale. Il s'agit ici d'une phase de planning qui doit permettre aux élèves de découvrir les documents sur lesquels ils vont travailler. Les élèves sont placés en binômes et préparent leur rôle en collaborant. Chaque paire prépare un des deux rôles: le rôle $\mathrm{A}$, les descendants d'immigrants et le rôle $\mathrm{B}$, les journalistes.

40 Les élèves sont regroupés en binômes qui se répartissent deux tâches. Les binômes $\mathrm{X}$ préparent le rôle de descendant d'immigrant (consigne : You found a diary written by your grandfather who emigrated to New York. Find the following information in the diary ${ }^{11}$. Les repérages à effectuer sont donnés sous forme de liste lexicalisée). L'objectif de cette première étape est de permettre aux élèves « interviewés » :

- de prendre connaissance du texte et se l'approprier ;

- de cibler les informations importantes à comprendre. Le travail s'effectue en binôme afin de favoriser l'entraide et de développer la motivation (incitation à participer);

- de réfléchir au sens du texte et à la manière de reformuler les informations-clé si nécessaire.

1 Les binômes $\mathrm{Y}$ préparent le rôle de journaliste (consigne: You want to write on immigration in New York for your school newspaper. You are going to interview an American immigrant's grandson/granddaughter who has discovered his/her grandfather's diary in which the details of his journey and arrival in New York are described. Prepare your questions ${ }^{12}$ ).

L'objectif de cette première étape est de permettre aux élèves « journalistes » :

- de préparer les questions qu'ils vont poser à partir de besoins spécifiques formulés en français sur une fiche préparée par l'enseignante. Il s'agira de former les questions en anglais ;

- de se préparer à expliciter leurs demandes, autrement dit d'être capable de reformuler leurs questions, si nécessaire.

Les schémas ci-dessous illustrent l'organisation de l'activité. 


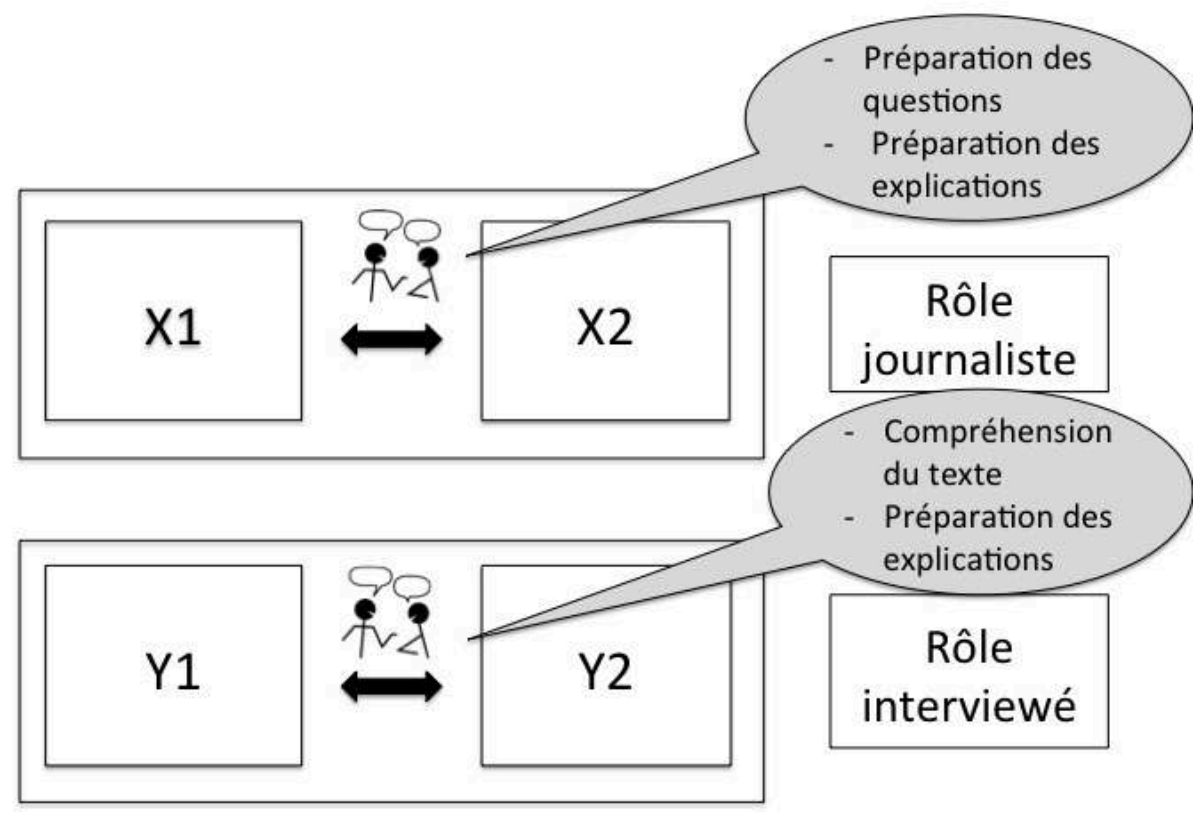

Figure 1 : Schéma d'organisation de l'étape 3 : préparation de l'interaction

\subsection{Pendant l'interaction : mise en place des stratégies de réussite}

Étape 4 : Il s'agit du jeu de rôle. Les élèves sont répartis dans de nouveaux binômes : X1 travaille avec Y1 et X2 avec Y2, etc. Des fiches d'observation sont fournies aux élèves pour qu'ils notent les stratégies de réussite mises en œuvre par les binômes en interaction. Il est demandé aux élèves de se focaliser sur plusieurs procédés : la reprise, la reformulation et l'explication ainsi que sur l'utilisation de gap fillers. Les stratégies de réussite propres à l'interaction orale, telles que les demandes, l'étayage ou de propositions d'aide, font partie des attendus qui doivent également être répertoriés. Le jeu de rôle est mis en œuvre de la façon suivante : pendant que X1 interviewe Y1, Y2 observe Y1 et X2 observe X1. X2 et Y2 prennent des notes sur leurs fiches d'observation. Puis les rôles sont inversés. 


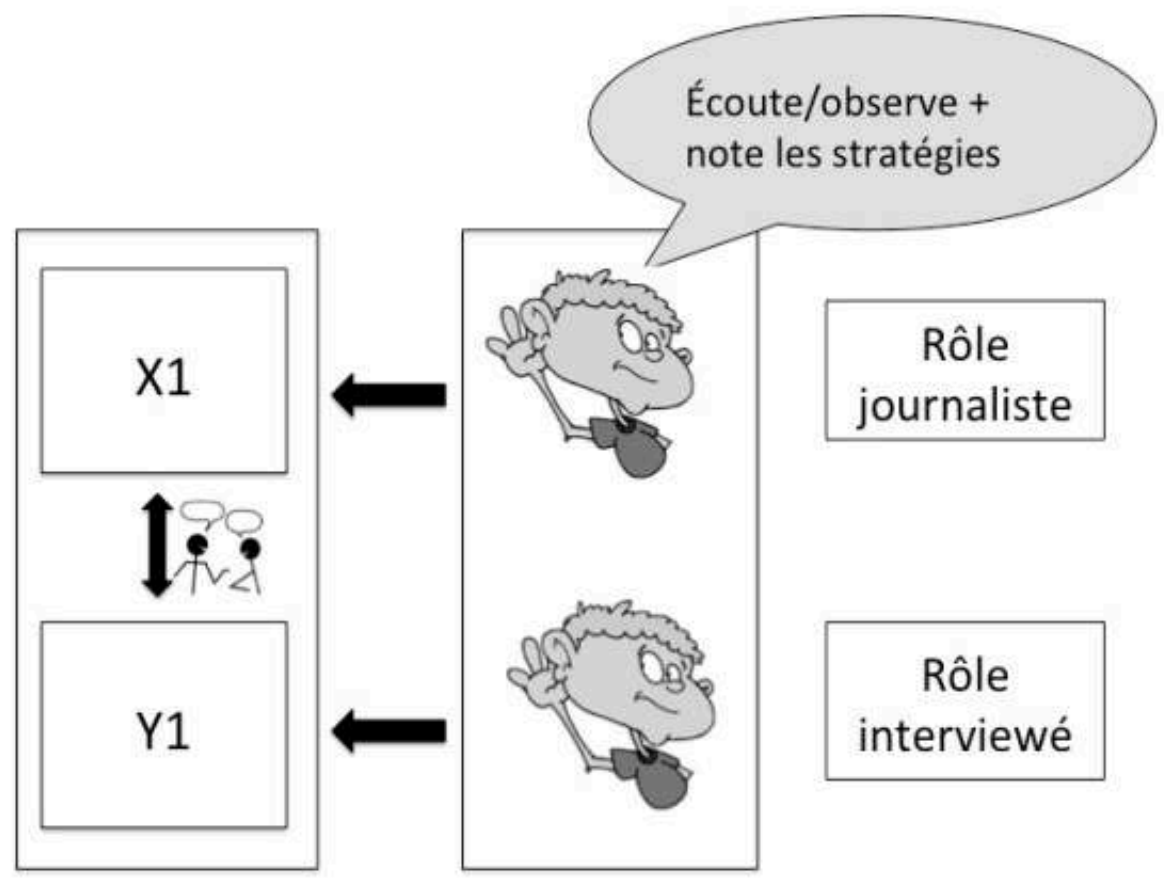

Figure 2 : Schéma d'organisation de l'étape 4, mise en œuvre de l'activité en interaction

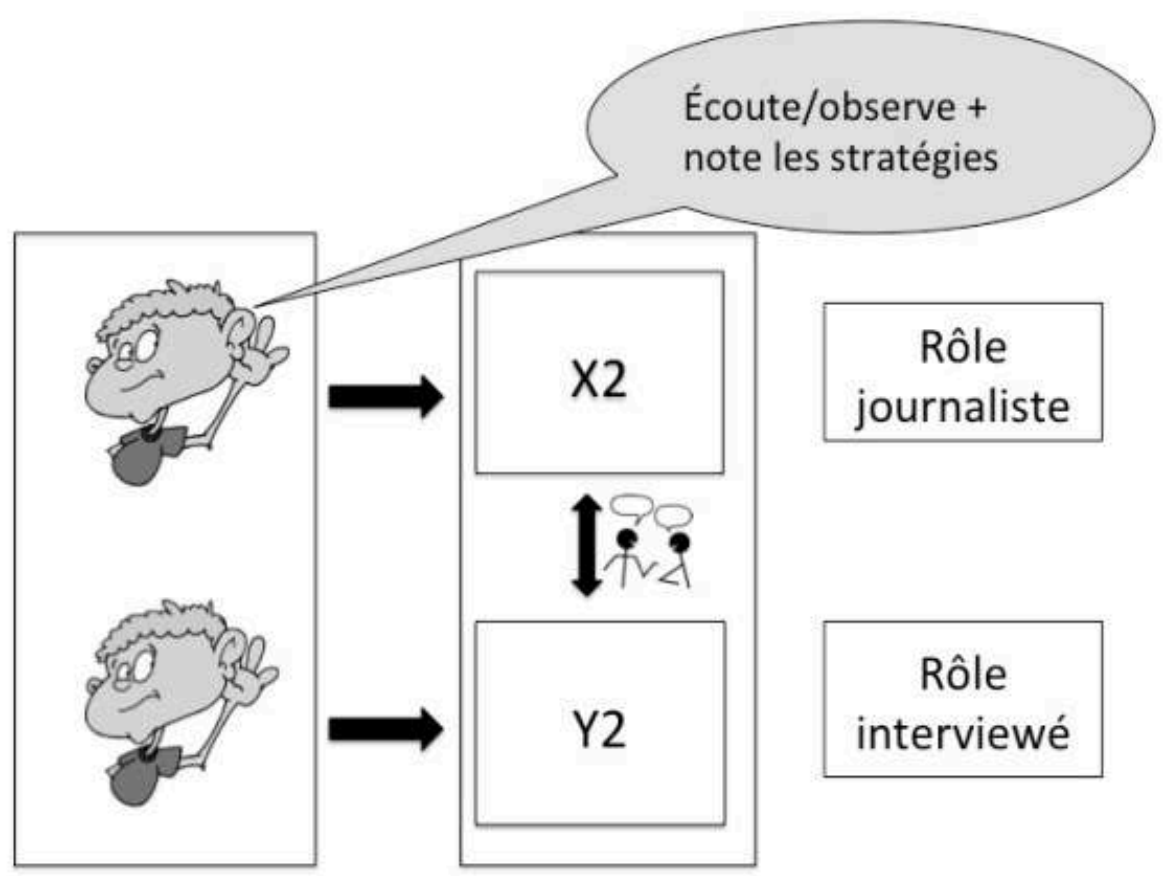

Figure 3 : Schéma d'organisation de l'étape 4, mise en œuvre de l'activité en interaction (suite) 


\subsection{Après : rétro-information sur les interactions}

Étape 5 : Une fois que tous les élèves ont terminé le jeu de rôle, chaque participant retourne avec son partenaire initial (X1 avec X2, etc.) pour un échange sur les stratégies observées dans l'interaction.

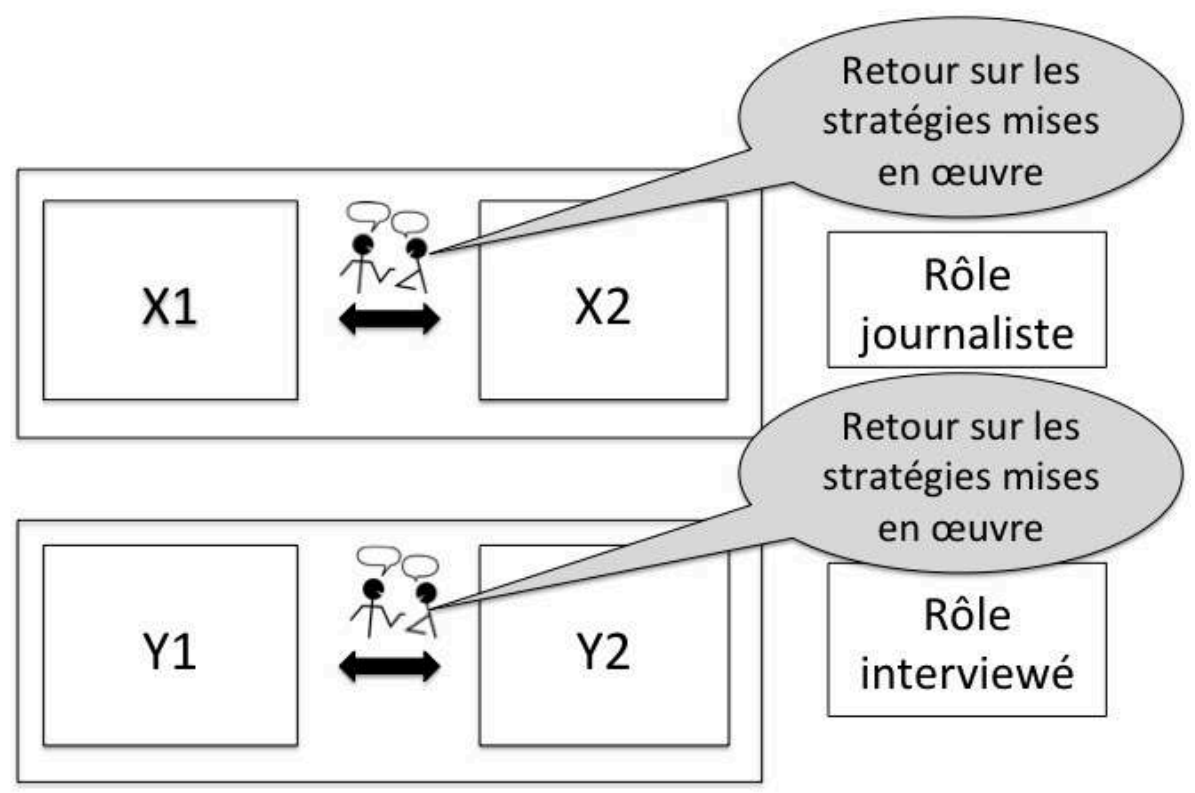

Figure 4 : Schéma d'organisation de l'étape 5 : rétro-information entre les partenaires

Sur la base des informations notées sur la fiche d'observations, les élèves commentent alors les réalisations de leurs partenaires.

Le protocole une fois établi, il nous est permis de d'émettre les hypothèses suivantes :

- interagir en langue étrangère n'est pas une compétence innée et nécessite un guidage ;

- ce guidage vise le développement d'une réflexion métacognitive et d'un processus d'autonomisation chez les apprenants ;

- l'autonomie envisagée s'inscrit dans une approche sociale reposant sur un apprentissage coopératif.

\section{Résultats}

Lors de cette expérimentation, la préparation (compréhension du document et rédaction des questions) a été menée lors d'une séance précédente. La réalisation de la tâche a pu être filmée pour quatre binômes et la phase de rétro-information pour deux binômes. Les transcriptions ont été réalisées sous CLAN (MacWhinney 2000), un logiciel permettant d'aligner la vidéo avec le texte et qui peut être enrichi d'annotations. 


\subsection{Les stratégies de reprise dans les interactions orales}

Pendant la réalisation de l'interview, les élèves ont véritablement incarné leur rôle, certains prenant très à cœur la dimension réaliste de leur attitude (séquence d'ouverture avec présentations et mains serrées) et/ou de l'authenticité des interactions langagières. Grâce à cet engagement, la plupart des problèmes de compréhension ou de production ont été traités par les participants lors de séquences de négociations du sens. Par ailleurs, plusieurs stratégies interactionnelles visant la progression du topique ont été utilisées, notamment lors du traitement des questions. Celles-ci sont décrites ci-dessous sous forme d'une typologie. On y voit apparaitre des reprises et des reformulations qui participent à la cohésion du discours et qui témoignent du contrôle que les participants exercent sur le discours-en-interaction lorsqu'ils sont engagés dans une tâche réalisée entre pairs et donc en autonomie.

\section{a. Reprise partielle ou totale de la question dans la réponse}

\section{- Pour maintenir la cohésion du discours}

Extrait 1

A09: What was the name of your grand-father?

A10: My grand-father's name was Isaac

Le format de la réponse (reprise presque intégrale des éléments de la question) est prototypique des échanges en la classe de langue. Dès les premières années de leur apprentissage, les élèves sont entrainés à reprendre les éléments de la question qui est gardée en mémoire. C'est un procédé peu exigeant au niveau cognitif qui garantit la cohésion et la cohérence des échanges.

\section{- Pour corriger une formulation incorrecte (dans une question)}

Extrait 2

A09: now did he live in New-York city?

A10: he still lives in New-York city.

com: hoche la tête

Ici, A10 propose une rétroaction corrective incorporée à sa réponse. C'est un moyen de rectifier discrètement la formulation précédente, sans paraître effectuer une correction. Le hochement de tête vient confirmer que le locuteur valide l'intercompréhension.

\section{- Pour demander une clarification}

B11 interroge C11 sur le terme «transatlantic liner » qui lui est inconnu. La reprise du terme permet de se focaliser sur la source du problème de communication et d'en faciliter la résolution.

Extrait 3

C11: he took a transatlantic liner.

B11: uh what is a transatlantic liner?

C11: it's a big ferry.

La préparation lors de l'étape préalable (phase de préparation) a permis à C11 d'élaborer une glose («a big ferry») pour ce terme qu'il ne connaissait probablement pas lui-même. Lorsque B11 interrompt la progression topicale pour introduire une 
séquence de négociation du sens, celle-ci est rapidement résolue grâce à la préparation qui lui permet de mobiliser rapidement une explication.

\section{- Pour gagner du temps}

\section{Extrait 4}

A11: what were his plans?

C12: plans of my grand-father are uh...+

C12: +, he gets married

C12 gagne ici du temps grâce à une reprise partielle de la question, placée en position initiale. Il manifeste malgré tout son appropriation du syntagme nominal en requalifiant l'expression référentielle « his » qui devient « my ». Cet épisode monologué à voix haute lui permet de conserver son tour de parole en informant son partenaire qu'une réponse est à venir. Celle-ci intervient effectivement après une pause sonore.

\section{b. Reprise écho}

\section{- Pour vérifier la compréhension}

\section{Extrait 5}

A09: uh (.) uh so what did he think about this trip?

A10: what do I [!] think?

A09: no what he [!] think.

A10 fait une reprise écho de la question qui lui a été posée pour vérifier sa compréhension. Son insistance (identifiée dans la transcription par [!]) sur le pronom sujet «I $I$ » est l'occasion de marquer l'item qui lui semble problématique, ce qui permet à $\mathrm{A} 09$ de rectifier en insistant à son tour sur le pronom « he ».

\section{- Pour gagner du temps et proposer une reformulation de la question}

Extrait 6

A09: and why uh he leave [/] uh he leaves Russia?

A10: uh why did he leave Russia?

A10: uh well, in Russia he lived in terrible poverty.

Après la formulation non canonique d'une question par A09 (et malgré une tentative d'autocorrection), le partenaire A10 propose une reprise avec reformulation. La vidéo permet de voir qu'il baisse la voix et que ses yeux décrochent de son partenaire pour regarder dans le vide, ce qui laisse supposer que la rétroaction corrective discrète n'est pas son objectif premier. La reprise prend une forme monologuée à voix haute qui lui permet de gagner du temps. Cette stratégie est confirmée par l'usage du marqueur discursif « ah well » placé au début de l'énoncé suivant qui retarde encore la réponse. La reprise du GN prépositionnel «in Russia » permet également de décaler l'introduction de l'information nouvelle qui apparaît en fin d'énoncé.

\section{c. Reformulation}

\section{- Pour aider}

Extrait 7

B12: and he have a [/] a chambre@s in the ferry?

B09: 0.

com: fronce les sourcils

B09: a room? 
B12: yes yes!

B09 est très investi dans le jeu de rôle et dans la mise en œuvre de la démarche méthodologique enseignée. Il s'attache donc à maintenir une stricte intercompréhension dans la langue cible en reformulant en anglais ce que B12 avait exprimé en français.

\section{- Pour vérifier la compréhension}

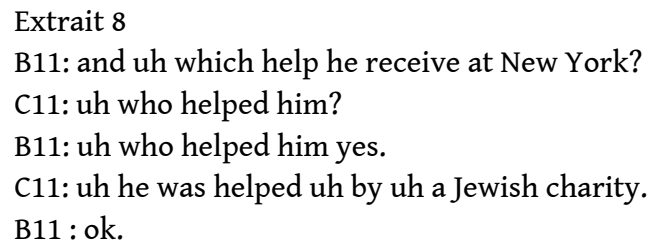

B11 calque la structure de sa question en anglais sur la consigne donnée en français dans sa fiche (tu veux savoir : l'aide qu'il a reçue à NY). C11 vérifie qu'il a bien compris ce qui lui est demandé en reformulant la question. Il peut également être raisonnable d'imaginer que cette reformulation est un moyen habile de corriger son partenaire. B11 confirme en effet la forme proposée par une reprise à l'identique et l'ajout d'un marqueur de validation « yes » à la fin de l'énoncé.

\subsection{Les réitérations comme outil de progression thématique}

D'autres stratégies telles que les demandes de répétitions et les reprises ont également été mises en œuvre pour résoudre les problèmes de communication. L'extrait cidessous met en lumière une séquence latérale dans laquelle la répétition (1.66) et la reprise (1.69) apparaissent comme des outils opérationnels pour faciliter l'intercompréhension et faire avancer le topique.

Extrait 9

$61 \mathrm{~B} 11:$ what he took between Ellis Island and NY?

62 C11: 0.

63 com: s'avance

64 B11: the mean of transport.

$65 \mathrm{C} 11:+<$ can you repeat?

$66 \mathrm{C} 11$ : the mean of transport.

67 B11: yes.

68 C11: uh I don't understand.

69 B11: you don't understand?

70 B11: at Ellis Island to NY.

71 C11: yes.

72 B11: uh he take the train?

73 C11: no a ferry.

74 B11: he take a ferry to Ellis Island at New-York?

75 C11: $+<$ to [/] to Manhattan.

76 B11: ah to Manhattan ok.

Outre ces stratégies de réitération, B11 propose un étayage (1.72) pour guider son partenaire qui ne comprend pas le syntagme nominal «the mean of transport» (la transcription orthographique est ici fidèle à la prononciation de l'élève). Cela met C11 sur la piste et lui permet de répondre à la question (1.73). L'étayage fait également parti $\mathrm{du}$ répertoire de stratégies enseigné dans la classe, que l'élève B11 s'est visiblement approprié. 
61

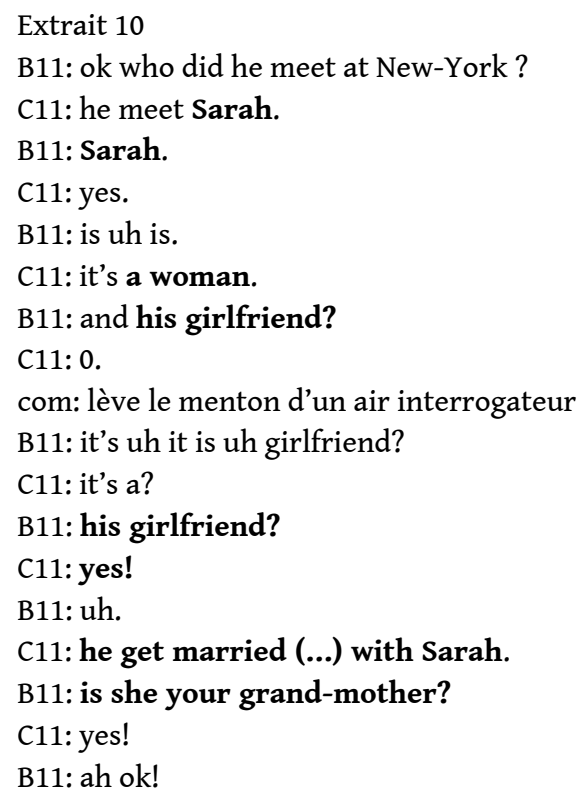

L'échange illustre bien la construction du sens du partagé : on observe la progression du prénom «Sarah» vers le GN «a woman » puis vers un GN précédé d'un adjectif possessif «his girlfriend » qui, à la suite du verbe «get » qui marque le changement d'état, suivi de l'adjectif « married », devient « your grand-mother ».

Les séquences filmées dans lesquelles les élèves accomplissent la tâche d'interview montre que le déficit d'information qui existe entre les partenaires de chaque binôme crée des situations où l'intercompréhension est gérée en temps réel, au cours de séquences latérales de négociation du sens (Jefferson 1972). Les participants mettent alors en place des stratégies de réussite en s'appuyant sur plusieurs procédés comme la reprise ou la reformulation des questions ou des réponses, enfin de vérifier leur intercompréhension mais également de produire une rétroaction corrective. Il s'avère que dans les séquences observées, la reprise est effectivement un format opératoire adapté au niveau des élèves de niveau A2. Quant à la reformulation, elle a une double fonction: elle permet d'expliquer (paraphrase, glose) ou bien de corriger. C'est d'ailleurs une forme plus élaborée qui requiert des compétences linguistiques et pragmatiques plus développées. Ce procédé conduit à une forme plus complexe de résolution des problèmes de communication. Enfin, toutes les formes de réitération, parfois alliées à l'usage de gap fillers, participent également à la mise en œuvre des stratégies de gain de temps qui sont indispensables pour permettre aux apprenants d'élaborer leur discours à la fois sur le fond et sur la forme. L'autonomie laissée aux participants leur permet de construire eux-mêmes le mode de progression de leur discours, ce qui autorise un traitement adapté des échanges. Un certain nombre de compétences langagières ne sont pas encore suffisamment procéduralisées pour que les choix énonciatifs s'opèrent inconsciemment comme en L1. L'usage de phases régulières de réflexion, qui aboutit souvent au succès de la communication, est sans doute largement favorisé par la situation de travail entre pairs. Ainsi l'autonomie d'action propre aux interactions entre pairs est susceptible de favoriser ces moments de réflexion qui permettent de régler les problèmes de communication. Dans le cadre d'un 
travail coopératif, l'apprenant peut en effet gérer son temps d'expression, ce qui n'est pas le cas lorsque le professeur et les autres élèves de la classe sont en attente de sa production. Le partenaire dans l'interaction fait alors preuve de coopération patiente ou au contraire active, dans le cas où il produit un étayage ou toute autre forme d'aide à son partenaire.

\subsection{La phase de rétro-information} du travail coopératif. Chaque observateur a proposé un retour sur les stratégies de communication dont il a pu être témoin, à partir des notes prises sur la grille fournie par l'enseignante. Le bilan des stratégies utilisées est assorti de commentaires sur la conduite de l'activité ainsi que d'une auto-évaluation. Les commentaires émis font apparaitre une mise en mots permettant de valoriser les réalisations et de pointer les non-réalisations, tout en les dédramatisant. Les échanges lors de cette étape se font entre pairs, toujours dans le cadre du travail coopératif. Ni les autres élèves de la classe, ni l'enseignant ne sont témoins des commentaires émis, la face des participants est donc davantage préservée. Cet aspect lié à l'organisation du travail en autonomie est fondamental pour les adolescents en pleine construction identitaire, qui sont souvent fragiles et sensibles. À cette question de l'âge vient s'ajouter l'instabilité linguistique de l'apprenant de LVE de niveau A2 car les faibles compétences en langue sont également susceptibles de fragiliser l'estime de soi. Apprendre à coopérer signifie également appréhender autrement les questions de gestion de la face positive ou négative (Brown and Levinson 1987). L'interaction orale est source de risques importants pour les participants car toute nouvelle intervention peut potentiellement devenir un problème qui met l'un des interlocuteurs en situation de perdre la face.

Il s'avère que cette phase de conscientisation a eu plusieurs conséquences au niveau du processus d'apprentissage. Tout d'abord, les élèves se savent observés par leurs pairs (et par la caméra) et s'impliquent donc potentiellement davantage pour faire bonne figure (Goffman 1955). La coopération se développe aussi plus largement car le travail réalisé en commun fait sens et ouvre la voie à une réflexion métacognitive coconstruite. Ensuite, la prise de notes réalisée pendant la phase d'observation des pairs permet un retour détaillé des actions mises en œuvre. Ce bilan des stratégies déployées favorise une prise de conscience des mouvements stratégiques élaborés dans l'interaction et adaptés à la situation. En effet, l'attention portée aux procédés mis en œuvre dans leurs échanges par les participants au jeu de rôle permet à l'observateur d'acquérir une compréhension fine des processus d'interaction orale. Enfin, la phase de rétro-information permet également de revenir sur les réussites ou les échecs des participants : les apprenants qui ont mis en place des stratégies efficaces pour gérer les échanges voient leurs efforts reconnus par leurs pairs. Les performances plus mitigées sont dédramatisées. La mise en mots des succès ou au contraire des actions non réalisées participe ainsi à l'apprentissage de la coopération. On remarque que le bilan est assez factuel dans un premier temps puis se poursuit souvent par un commentaire appréciatif du type "c'est bien». Le discours des élèves se caractérise par un recours assez systématique à des formules d'atténuation lors de leurs commentaires sur les actions non-réalisées, avec des expressions comme "c'est pas grave». Certaines remarques relèvent du conseil tel que : « il faudrait utiliser plus de gap fillers quand tu ne sais pas quoi dire ». Par ailleurs, les élèves à qui sont adressés les commentaires ont 
également la possibilité de "s'expliquer » et ainsi de faire émerger les difficultés qu'ils ont rencontré ou encore les succès dont ils sont fiers. Cette verbalisation correspond à une prise de conscience de leur compétence et peut être le préalable à une recherche de solutions ou à la fixation d'objectifs d'apprentissage. Le discours de l'une des participantes témoigne assez clairement de l'importance des représentations qu'ont les apprenants de leurs savoirs et savoir-faire ainsi que du rôle que celles-ci jouent sur le développement de leurs compétences. L'élève $\mathrm{C} 12$ insiste dans son discours sur le bon niveau de sa partenaire qu'elle compare au sien, le considérant comme nettement inférieur. Clément (1980, cité par Dörnyei 2005 : 73) présente ce manque de confiance en soi comme une construction sociale engendrée par la représentation que les apprenants ont de leur niveau au sein d'une communauté. Ce positionnement comme apprenant peu compétent, même s'il est peu valorisant, peut être l'occasion d'identifier les difficultés rencontrées. On peut postuler que le retour de la camarade, qui a mis en avant ses réussites et a minimisé ses maladresses ou faiblesses, peut contribuer à restaurer une image plus positive ou, à défaut, de lui permettre de se fixer des objectifs à atteindre.

\section{Conclusion}

Le protocole décrit ici a permis d'observer au plus près les interactions orales entre pairs dans une classe de langue, ainsi que la coopération mise en place entre les participants. Compte-tenu des difficultés d'organisation pour filmer, en milieu écologique, des apprenants durant leurs activités de classe, l'échantillon est très restreint. L'analyse n'a donc qu'un caractère qualitatif qui vise à mettre en lumière les processus à l'œuvre. Néanmoins, les échanges enregistrés durant la phase de préparation ainsi que pendant la phase de rétro-information témoignent de l'importance du guidage, à la fois pour anticiper sur la tâche puis pour expérimenter les stratégies proposées et enfin pour conscientiser les réussites et les potentiels de développement. L'hypothèse formulée selon laquelle un guidage doit être mis en place pour former les apprenants à l'interaction orale en LVE s'avère dans les cas observés valide, puisque les participants ont réinvesti les stratégies enseignées et ont largement participé à la réussite des échanges.

67 Le dispositif mis en place emprunte à la fois à l'apprentissage coopératif et à l'apprentissage collaboratif. Les spécificités liées au travail en LVE en milieu institutionnel exolingue impose la rigueur de l'organisation des groupes coopératifs et la répartition des activités, tout en adoptant les principes de symétrie et d'équilibre qui sont essentiels dans l'apprentissage collaboratif pour libérer la parole. L'expérimentation décrite ainsi que toutes celles observées au cours de l'année scolaire ont permis, aux dires de leur enseignante, de responsabiliser les élèves dans leurs modes d'apprentissage. En effet, leur attitude dans les activités d'interaction orale a rapidement évolué avec une mise au travail rapide et des échanges réalisés quasi exclusivement en anglais, y compris lorsque des problèmes de communication apparaissaient. Leur degré d'autonomie dans la réalisation des travaux de classe a donc progressé, comme cela avait été envisagé. Par ailleurs, les élèves ont développé une connaissance avérée des stratégies propres à faciliter le discours-en-interaction (Manoïlov 2017). Ces observations ont été l'occasion de faire émerger la manière dont les élèves se sont approprié les stratégies d'interaction orale qu'ils avaient découvertes 
au préalable. Enfin, la phase de conscientisation leur a semble-t-il permis de mener une réflexion sur leurs performances susceptibles de favoriser un processus d'autorégulation. La conscientisation et la verbalisation de stratégies métacognitives contribuent ainsi à une plus grande responsabilisation des apprenants qui peuvent alors se fixer des objectifs d'apprentissages répondant à leurs besoins propres. C'est d'ailleurs l'approche développée par le Cadre Européen de Référence pour les Langues (Conseil de l'Europe 2001, 2018) qui propose des grilles d'auto-évaluation des savoirfaire formulés à l'aide d'énoncés à la première personne du singulier. Ce type de dispositif est ainsi susceptible de favoriser une individualisation des apprentissages qui ne sont plus, dans cette optique, considérés comme linéaires et émanant de l'enseignant mais au contraire organiques car construits consciemment par chaque individu au gré des rencontres, des échecs et des réussites.

\section{BIBLIOGRAPHIE}

ARONSON, E., BLANEY, N., STEPHAN, C., SIKES, J., SNAPP, M., 1978. The jigsaw classroom, Beverly Hills, California: Sage Publications.

BANDURA, A., 1989, « Human Agency in Social Cognitive Theory », American Psychologist, 44(9), p. 1175-1184.

BANGE, P., 2005, L'apprentissage d'une langue étrangère, Paris : L'Harmattan.

BARBOT, M. J., CAMATARRI, G., 1999, Autonomie et apprentissage. L'innovation dans la formation, Paris : PUF.

BAUDRIT, A., 2007, « Apprentissage coopératif/Apprentissage collaboratif : D’un comparatisme conventionnel à un comparatisme critique », Les Sciences de l'éducation - Pour l'Ere nouvelle 40(1), p. 115-136.

BERGER, E., 2008, « La reprise comme ressource interactionnelle en langue seconde », Travaux Neuchâtelois de Linguistique 48, p. 43-61.

BIANCO, M., 2016, Lire pour comprendre et apprendre: Quoi de neuf? Lyon : CNESCO-IFÉ.

BROWN, P., LEVINSON, S. C., 1987, Politeness: Some universals in language usage, Cambridge:

Cambridge University Press.

BRUNER, J., 1983, Le développement de l'enfant ; savoir faire, savoir dire, Paris : PUF.

CALDERÓN, M., SLAVIN, R., SÁNCHEZ, M., 2011, Effective Instruction for English Learners, Future of Children 21(1), p. 103-127.

COHEN, A. D., WEAVER, S. J., 1998, « Strategies-based instruction for second language learners ", dans W.A. Reyandya \& G.M. Jacobs Learners and Language Learning, Singapore : SEAMEO Regional Language Center, p. 1-25.

CONSEIL DE L'EUROPE, 2001, Cadre Européen Commun de Référence pour les Langues-Apprendre, Enseigner, Evaluer, Paris : Didier. 
CONSEIL DE L'EUROPE, 2018, Cadre Européen Commun De Référence Pour Les Langues: Apprendre, Enseigner, Evaluer Volume Complémentaire avec de Nouveaux Descripteurs.

DAMON, W., 1984, « Peer education: The untapped potential », Journal of Applied Developmental Psychology 5(4), p. 331-343.

DEWEY, J., 1933, How We Think: A Restatement of the Relation of Reflective Thinking to the Educative Process, Boston, MA: D.C. Heath \& Co Publishers.

DILLENBOURG, P., 1999, « What do you mean by 'collaborative learning'? », dans P. Dillenbourg (ed.), Collaborative learning: Cognitive and computational approaches, Oxford: Pergamon Press, p. 1-19. DOISE, W., MUGNY, G., 1981, Le développement social de l'intelligence, Paris : Inter Editions. DÖRNYEI, Z., 1995, « On the Teachability of Communication Strategies », TESOL Quarterly 29(1), p. 55-85.

DÖRNYEI, Z., 2005, The Psychology of the Language Learner, Erlbaum.

EHRMAN, M. E., DÖRNYEI, Z., 1998, Interpersonal Dynamics in Second Language Education. The Visible and Invisible Classroom, London: Sage Publications.

ELLIS, R., 2003, Task-based Language Learning and Teaching, Oxford: Oxford University Press.

GOFFMAN, E., 1955, « On Face-Work: An analysis of ritual elements in social interaction », Psychiatry: Journal of Interpersonal Relations 18(3), 213-231.

GOODWIN, C., 2017, Co-Operative Action. Cambridge University Press.

GRIST, M., 2009, Changing the Subject: how new ways of thinking about human behavior might change politics, policy and practice, London: Royal Society of Arts, Retrieved from: [https:// www.thersa.org/globalassets/pdfs/blogs/nov28th2009changingthe-subjectpamphlet.pdf]

HILTON, H., 2019, Sciences cognitives et apprentissage des langues [Rapport scientifique d'une Conférence de consensus], Paris : CNESCO.

HOLEC, H., 1979, Autonomie et Apprentissage Des Langues Étrangères, Paris : Hatier.

HOLEC, H., 1990, « Qu'est-Ce Qu'apprendre à Apprendre? », Mélanges Pédagogiques, p. 75-87.

HOLEC, H., 1991, « Autonomie de l'apprenant : De l'enseignement à l'apprentissage », Education Permanente 107, p. 59-66.

HOWE, C., 2010, Peer groups and children's development. Oxford: Wiley-Blackwell.

JEFFERSON, G., 1972, « Side sequences », dans D.N. Sudnow (ed.) Studies in social interaction, New York: Free Press, p. 294-338.

JÉZÉGOU, A., 2013, « The influence on the openness of an e-learning situation on adult- students' self-regulation ", International Review of Research on Open and Distance Learning 14(3), p. 182-201.

JOHNSON, D. W., JOHNSON, R. T., 1975, Learning together and alone: Cooperation, competition and individualization, Englewood Cliffs, NJ: Prentice-Hall.

JOHNSON, D. W. and JOHNSON, R. T., 1990, « What is cooperative learning? ", dans M. Brubacher, R. Payne, K. Rickett (ed.) Perspectives on small group learning, Oakville: Rubicon Publishing Inc., p. 68-80.

JOHNSON, D. W., JOHNSON, R. T., 1998, Learning together and alone: Cooperative, competitive, and individualistic learning (5th ed.), Boston: Allyn \& Bacon. 
LAZARATON, A., DAVIS, L., 2008, « A Microanalytic Perspective on Discourse Proficiency, and Identity in Paired Oral Assessment », Language Assessment Quarterly 5(4), p. 313-335.

LONG, M., PORTER, P., 1985, « Group work, interlanguage talk, and second language acquisition », TESOL Quarterly 19, p. 207-225.

MACARO, E., 2001, Learning strategies in second and foreign language classrooms, London: Continuum.

MACWHINNEY, B., 2000, The CHILDES Project: Tools for Analyzing Talk, Mahwah, NJ: Erlbaum.

MANOÏLOV, P., 2017, L'interaction orale entre pairs en classe d'anglais LV2. Analyse didactique et linguistique de la construction et du développement des compétences des apprenants, Sorbonne Nouvelle-Paris 3, Paris.

MANOÏLOV, P., 2019, « Repenser l'organisation des tâches pour favoriser le développement des interactions orales entre pairs », Les Langues Modernes 3, p. 18-27.

MANOÏLOV, P., OURSEL, É., 2020, « Analyse des interactions et didactique des langues: Tour d'horizon des relations », Linx. Revue Des Linguistes de l'université Paris Nanterre.

MERCER, N., 2016, « Education and the Social Brain: Linking Language, Thinking, Teaching and Learning », Éducation et Didactique 10(2), p. 9-23.

MINISTÈRE DE L'ÉDUCATION NATIONALE ET DE LA JEUNESSE, 2019, Repères et références statistiques, DEPP.

NAKATANI, Y., 2005, « The Effects of Awareness-Raising Training on Oral Communication Strategy Use », The Modern Language Journal 89(i), p. 76-91.

NAKATANI, Y., 2010, « Identifying strategies that facilitate EFL learner's oral communication: A classroom study using multiple data collection procedures ", The Modern Language Journal 94(i), p. 116-136.

PANITZ, T., 1999, Cooperative versus collaborative learning: A comparison of two concepts which will help us understand underlying nature of interactive learning, US Department of Education.

PEKAREK DOEHLER, S., DE PIETRO, J.-F., FASEL LAUZON, V., POCHON-BERGER, E., 2008, L'organisation du discours dans l'interaction en langue première et seconde: Acquisition, enseignement, évaluation [Rapport final Projet CODI], Neuchâtel : Université de Neuchâtel \& IRDP.

PERRET-CLERMONT, A.-N., 1979, La construction de l'intelligence dans l'interaction sociale, Genève : Peter Lang.

PHILP, J., ADAMS, R., IWASHITA, N., 2014, Peer Interaction and Second Language Learning, New York: Routledge.

PICA, T., LINCOLN-PORTER, F., PANINOS, D., LINNEL, J., 1996, TESOL Quaterly 30 (1), p. 59-83.

PRABHU, N. S., 1987, Second Language Pedagogy, Oxford: Oxford University Press.

RIVENS MOMPEAN, A., 2013, Le Centre de Ressource en Langues: Vers la modélisation du dispostif d'apprentissage, Villeneuve d'Ascq : Septentrion.

ROUSSEL, S., 2019, Les activités cognitives de l'apprenant en classe de langue: L'exemple de la compréhension de l'oral, Retrieved from https://www.dailymotion.com/video/x7598ba.

SLAVIN, R. E., 1996, « Research on Cooperative Learning and Achievement: What We Know, What We Need to Know », Contemporary Educational Psychology 21(1), p. 43-69.

TARONE, E., 1980, « Communication strategies, foreigner talk, and repair in interlanguage studies ", Language Learning 30, p. 417-431. 
TOBIAS, S., EVERSON, H. T., 2009, « The Importance of Knowing What You Know. A Knowledge Monitoring Framework for Studying Metacognition in Education », dans D. J. Hacker, J. Dunlosky, A. C. Graesser (ed.) Handbook of metacognition in education, New York: Routledge, p. 107-128.

VAN LIER, L., 1996, Interaction in the Language Curriculum: Awarness, Autonomy \& Authenticity, New York: Routledge.

VANDERGRIFT, L., GOH, C., 2012, Teaching and learning second language listening: Metacognition in action, New York: Routledge.

VYGOTSKY, L. S., 1978, Mind in Society, Cambride, Mass.: Harvard University Press.

WHITBREAD, D., MERCER, N., HOWE, C., TOLMIE, A., 2013, Self-regulation and dialogue in primary classrooms, Leicester: British Psychological Society.

WITTROCK, M. C., 1986, « Students' thought processes », dans M. C. Wittrock (ed.) Handbook of Research on Teaching (3rd ed.), New York: Macmillan, p. 297-314.

\section{NOTES}

1. C'est d'ailleurs l'un des problèmes du CECRL (Conseil de l'Europe 2001) dont la cible initiale est davantage l'apprenant adulte. Les nouveaux descripteurs du volume complémentaires (2018) traitent ce problème en indiquant la pertinence des capacités décrites pour le groupe d'âge des 11-15 ans.

2. " any communicative activity carried out between learners, where there is minimal or no participation from the teacher (notre traduction)

3. Jodelet (1980) indique que les facteurs socio-affectifs relèvent de l'implication émotionnelle vis-à-vis de l'entourage social et de la signification sociale accordée à l'action réalisée.

4. Le travail avec un pair, en binôme (ou en paire) est ici considéré comme du travail en groupe, un groupe composé de 2 personnes.

5. Le besoin d'autonomie (c'est-à-dire le désir d'initier et de réguler ses actions) est un besoin humain inné. Un certain niveau d'autonomie est considéré comme une condition préalable pour que tout comportement soit intrinsèquement gratifiant (notre traduction).

6. Pour Holec, le concept d'autonomie chez l'apprenant a des conséquences non seulement sur la manière dont l'apprentissage est organisé, mais aussi sur le type de connaissances acquises. Si l'apprenant détermine lui-même les buts et les contenus d'apprentissage, 'le concept de connaissance objective et universelle est remplacé par une connaissance subjective et individuelle'. (Notre traduction)

7. Des individus [qui] prennent l'initiative, avec ou sans l'aide des autres, de diagnostiquer leurs besoins d'apprentissage, de formuler leurs objectifs d'apprentissage, d'identifier les ressources humaines et matérielles nécessaires pour soutenir leur apprentissage, de choisir et de mettre en œuvre des stratégies d'apprentissage appropriées et d'évaluer les résultats de ces apprentissages. (Notre traduction)

8. one's ability to monitor, evaluate, and make plans for one's learning. (Notre traduction)

9. «la coordination médiée par le langage de l'activité collective implique que les individus soient capables d'utiliser le langage comme moyen d'organiser et de contrôler leurs propres actions et leur comportement au cours de la tâche commune. » (Notre traduction).

10. Bulletin officiel spécial $n^{\circ} 11$ du 26 novembre 2015

11. « Tu as découvert le journal intime de ton père qui a émigré à New York. Trouve les informations suivantes dans son journal. » (Notre traduction). 
12. «Tu veux écrire un article sur l'immigration dans le journal du collège. Pour cela, tu vas interviewer le petit-fils/la petite-fille d'un immigrant qui a découvert le journal intime de son grand-père et dans lequel on peut lire le récit détaillé de son voyage et de son arrivée à New York. Prépare tes questions. » (Notre traduction).

\section{RÉSUMÉS}

Cet article rend compte d'une approche développée en classe d'anglais au collège en France afin de former les apprenants à coopérer pour apprendre mais également, à apprendre à coopérer. Le postulat développé ici est que c'est dans et par l'usage que les formes linguistiques émergent et que se développe le processus dynamique d'acquisition. Dans cette optique, les échanges entre pairs en classe de langue permettent de maximiser les temps d'échange et la pratique qui en découle est susceptible de venir soutenir le développement des apprentissages. Néanmoins, la coopération entre apprenants ne peut être considérée comme acquise a priori, ni comme une aptitude maitrisée par les élèves au collège. Dans la recherche-action présentée ici, les apprenants ont bénéficié d'une formation à certaines stratégies propres à l'interaction orale et ont été sensibilisés à la dimension collaborative de l'apprentissage. Afin de réaliser une tâche, les élèves se sont engagés dans des interactions orales qui ont été observées par des pairs. Les participants ont pu ensuite échanger sur les stratégies collaboratives mises en place pour résoudre les problèmes de communication et prendre conscience de leurs réussites et de leurs besoins.

This article reports on an approach developed in EFL classes in secondary schools in France to train learners to cooperate in learning, but also to learn to cooperate. The premise developed here, and shared by many researchers in language acquisition/learning, is that it is in and through the use of language that linguistic forms emerge and that the dynamic process of acquisition develops. In this perspective, peer interaction in language classes makes it possible to maximize exchange times and the ensuing practice is likely to support the development of learning. Nevertheless, willing to cooperate or being able to cooperate are not abilities that can be considered as acquired by middle school students. In this action research, learners were trained to develop some strategies that are specific to spoken interaction and to appreciate the collaborative dimension of learning. Using a task-based learning approach, students were set to engage in spoken interactions while they were observed by peers. The participants were then able to share their collaborative strategies to solve communication problems and become aware of their successes and needs.

\section{INDEX}

Mots-clés : interaction entre pairs, coopération, collaboration, apprentissage et enseignement des langues

Keywords : peer interaction, cooperation, collaboration, SLLT 
AUTEUR

PASCALE MANOÏLOV

Maitre de conférences, Université Paris 10 - Nanterre 